\title{
Rhetorik gegen Spekulation Ein Antagonismus der scholastischen Bildungsgeschichte
}

\author{
Catherine König-Pralong (Freiburg im Breisgau)
}

„This also must be confessed, that the most durable, as well as justest fame, has been acquired by the easy philosophy, and that abstract reasoners seem hitherto to have enjoyed only a momentary reputation, from the caprice or ignorance of their own age $[\ldots]$. The fame of Cicero flourishes at present; but that of Aristotle is utterly decayed."

David Hume, An Enquiry concerning Human Understanding, I, 4, Oxford 2007, 4.

In einer Schrift aus dem Jahre 1868 diagnostiziert Ernest Renan den Verfall der französischen Universität, den er mit dem wissenschaftlichen Aufschwung der deutschen Universität kontrastiert ${ }^{1}$ :

„L'Université de France [...] rappelle trop les rhéteurs de la décadence. Le mal français, qui est le besoin de pérorer [...] une partie de l'Université l'entretient par son obstination à mépriser le fond des connaissances et à n'estimer que le style et le talent.“2

Renan zufolge besteht der Mangel der französischen Universität im rhetorischen Charakter ihres Unterrichts. Renan inszeniert eine Auseinandersetzung zwischen der von ihm diagnostizierten Oberflächlichkeit des französischen Wis-

1 Mein Dank gilt Nadja Germann für die sprachliche Korrektur dieses Textes sowie für ihre Anmerkungen zum Inhalt.

Die vorliegende Untersuchung ist Teil des ERC-2013-CoG Projekts MEMOPHI (Medieval Philosophy in Modern History of Philosophy).

2 E. Renan, Questions contemporaines, Paris ${ }^{2} 1968$, V. Renan führt eine geschichtliche Erklärung an: Die Vertreibung der Protestanten aus Frankreich hat das französische Studium beschädigt: „La France protestante était en train de faire, dans la première moitié du XVII ${ }^{\mathrm{e}}$ siècle, ce que l'Allemagne protestante fit dans la seconde moitié du XVIII ${ }^{\mathrm{e}}$ siècle. Il en résultait pour tout le pays un admirable mouvement de discussion et de recherches. [...] La révocation de l'Édit de Nantes brisa tout cela. Elle tua les études de critique historique en France. L'esprit littéraire étant seul encouragé, il en résulta une certaine frivolité." (Ibid., 80). Zu diesem Text und zu Renans Stellungnahme: P. Bourdieu, Systèmes d'enseignement et systèmes de pensée, in: F. Clément e. a. (eds.), L’inconscient académique, Genève-Zürich 2006, 21-46. 
sens und der Ernsthaftigkeit der protestantischen Gelehrsamkeit. Gegenstand meines Beitrags ist die Untersuchung der geschichtlichen Konstitution dieses für die europäische Kultur prägenden und noch heute vorherrschenden Antagonismus zwischen Rhetorik und universitärem Wissen.

Der Verdacht, der der Rhetorik an den abendländischen Universitäten wiederkehrend entgegengebracht wurde, stellt keine Selbstverständlichkeit der Bildungsgeschichte dar. In philosophiegeschichtlicher Hinsicht ist die antirhetorische Einstellung nicht das einzige Modell der guten wissenschaftlichen Praxis; sie stemmte sich gegen konkurrierende Wissenskonzeptionen und setzte sich in der hier relevanten Form erst am Ende des Mittelalters durch. Die Inszenierung eines Spannungsfelds zwischen zwei Philosophiekonzeptionen anhand des Kriteriums ihrer rhetorischen Prägung wurde zwar schon im 4. Jahrhundert vor Christus in den Debatten der konkurrierenden Schulen von Isokrates und Platon geschildert ${ }^{3}$; eine starke und bewusste Verbannung der Rhetorik aus der Philosophie erfolgte aber, meiner Einschätzung nach, erst im 13. Jahrhundert. Sie hängt mit einer bestimmten Institution, der neu gegründeten Universität zusammen. Demzufolge ist der wissenschaftliche Verdacht gegen die Rhetorik als ein Ereignis der Philosophie- und Kulturgeschichte zu betrachten und zu erforschen. Im Unterschied zum Versuch von Alex Novikoff, die Kontinuität der Wissenspraktiken vom antiken Dialog zur scholastischen disputatio am Beispiel der Dialoge des Anselm von Canterbury zu beweisen ${ }^{4}$, lenke ich die Aufmerksamkeit auf ein Moment der Bildungsgeschichte, um die Spezifizität der universitären Wissenspraktiken hervorzuheben.

\section{Die universitäre Wende}

Vom ersten Jahrhundert vor Christus bis zum 13. Jahrhundert ist der Antagonismus zwischen wissenschaftlicher Ernsthaftigkeit und rhetorischer Brillanz kaum zu belegen. In der römischen Antike, mindestens bis zur Zeit Augustinus' und Hieronymus', bildete die ciceronianische Rhetorik die Grundlage der philosophischen Ausbildung, wenn nicht der Bildung überhaupt. Hatte sich schon das von Platon im ,Phaidros' entworfene rhetorische Ideal des Isokrates gegen Platon selbst, aber auch gegen Aristoteles und die Stoiker durchgesetzt, so identifizierte im 1. Jahrhundert nach Christus Quintilian den Orator mit dem Schola-

3 Cf. W. L. Benoit, Isocrates and Plato on Rhetoric and Rhetorical Education, in: Rhetoric Society Quarterly 21 (1991), 60-71; P. Demont, Isocrates et le Gorgias de Platon, in: L'Information littéraire 60 (2008), 3-9.

4 A. Novikoff, The Medieval Culture of Disputation. Pedagogy, Practice, and Performance (The Middle Ages Series), Philadelphia 2013, 35: „This chapter aims to show that the scholastic dialectical methods, later so prominent in medieval universities, have their origins within the general milieu of monastic learning. More specifically, these methods will be shown to have their origins in Lanfranc's and Anselm's engagement with dialogue and disputation at the school of Bec." 
sticus. Schule und Rhetorik bildeten die Form und den Inhalt einer einheitlichen Wissensinstitution. Dementsprechend verweist im klassischen Latein das Adjektiv ,scholasticus" auf den Rhetorikunterricht, während das Substantiv „scholasticus" den Studenten bezeichnet, der die Rhetorikklasse besucht ${ }^{5}$. Im 8. Jahrhundert verfasste Alkuin einen Dialog, De arte rhetorica' für den Gebrauch in der Schule $^{6}$. Eine enge Assoziation der Rhetorik nicht nur mit den Rechts- und Politikwissenschaften, sondern auch mit der Philosophie und der Theologie lässt sich darüber hinaus bis zum 12. Jahrhundert u. a. bei Peter Abaelard, Johannes von Salisbury und der Schule von Sankt Viktor bezeugen ${ }^{7}$.

In der sogenannten ,scholastischen“ Zeit an der mittelalterlichen Universität veränderte sich die intellektuelle Konstellation jedoch radikal. Als Bezeichnung für einen Schüler oder Magister des 13. Jahrhunderts erhält der philosophiegeschichtliche Begriff „Scholastiker“ eine neue, ganz unterschiedliche Bedeutung. Diesen Wandel veranschaulicht die Geschichte des Scholasticus-Konzepts, die vom evangelischen Polemiker Adam Tribbechow 1665 veröffentlicht und von Jacob Brucker ${ }^{8}$ und der aufklärerischen Philosophiegeschichtsschreibung im 18. Jahrhundert übernommen wurde. Während der antike Scholastiker ein gebildeter Redner war, haben die barbarischen Scholastiker des Mittelalters die Rhetorik vernachlässigt, verdorben und verachtet: „[...] eloquentiae hostes Scholastici, Sophisticen contra amplexi, ea foeditate quidvis coinquinare unice studuerunt." "9

Zwanzig Jahre vor seinen ersten Studien zur Medientheorie, genauer 1943, hatte der berühmte Medienforscher Marshall McLuhan seine Dissertation ge-

5 Cf. R. Quinto, Scholastica. Storia di un concetto (Subsidia mediaevalia Patavina 2), Padova 2001, 34-36.

6 Cf. W. Hartmann, Rhetorik und Dialektik in der Streitschriftenliteratur des 11./12. Jahrhunderts, in: J. Fried (ed.), Dialektik und Rhetorik im früheren und hohen Mittelalter. Rezeption, Überlieferung und gesellschaftliche Wirkung antiker Gelehrsamkeit vornehmlich im 9. und 12. Jahrhundert (Schriften des Historischen Kollegs 27), München 1997, 73-96, hier 78.

7 Über Abaelard und den rhetorischen Charakter seiner Lehre sowie seines Werkes: P. von Moos, Rhetorik, Dialektik und ,civilis scientia' im Hochmittelalter, in: Fried (ed.), Dialektik und Rhetorik (nt. 6), 135-156; P. von Moos, Literary Aesthetics in the Latin Middle Ages: The Rhetorical Theology of Peter Abelard, in: C. J. Mews/C. J. Nederman/R. M. Thomson (eds.), Rhetoric and Renewal in the Latin West 1100-1540. Essays in Honour of John Ward (Disputatio 2), Turnhout 2003, 55-80. Im selben Band begegnet der Leser jedoch einer abweichenden Darstellung des philosophischen und theologischen Projekts von Abaelard. Constant J. Mews betrachtet Abaelard nämlich als einen Dialektiker, der die Überlegenheit der Dialektik über die Grammatik und die Rhetorik verteidigte (C. J. Mews, Peter Abelard on Dialectic, Rhetoric and the Principles of Argument, ibid., 37-53). Bereits 1943 zeigte jedoch Marshall McLuhan in seiner (erst 2006 veröffentlichten) Dissertation, dass Abaelard, der Verteidiger der Dialektik, oft als reiner Grammatiker verfährt (M. McLuhan, The Classical Trivium. The Place of Thomas Nashe in the Learning of his Time, Corte Madera 2006 [1943], 103 sqq.). Darüber hinaus dokumentierte McLuhan die Nachhaltigkeit der Grammatik und der Rhetorik und deren große Bedeutung in der Schule von Sankt Viktor (ibid., 103-105). Das ,Metalogicon' des Johannes von Salisbury las er als eine Verteidigung der ciceronianischen Rhetorik (ibid., 147-149).

8 Jacob Brucker, Historia Critica Philosophiae, Leipzig 1742-1744, ${ }^{2} 1766-1767$, vol. 3, 709714.

9 Adam Tribbechow, Liber de doctoribus scholasticis (Zweite Ausgabe mit einem Vorwort von Christoph August Heumann), Jena 1665, ${ }^{2} 1719,41$. 
rade diesem Thema gewidmet ${ }^{10}$. Gegen die verbreitete Ansicht, die Renaissance bedeute ein Wiederbeleben der im Mittelalter vergessenen „Belles Lettres“, hat McLuhan das Fortdauern der „Belles Lettres“, der Rhetorik und der Grammatik während des gesamten Mittelalters nachgewiesen. In diesem Zusammenhang stellt die universitäre Kultur des 13. Jahrhunderts eher einen Wendepunkt sowie eine Ausnahme dar als den Inbegriff der mittelalterlichen Kultur. Sie setzt einen neuen wissenschaftlichen Stil durch, der sich von der Redekunst eindeutig abgrenzt.

Meistens erklären die Philosophiehistoriker diesen Wandel durch das Erbe des vollständigen aristotelischen Corpus. Die Aneignung der demonstrativen Methode der ,Zweiten Analytiken“ sowie die Rezeption der aristotelischen Metaphysik, Psychologie und Naturphilosophie haben die philosophische Landschaft grundlegend verändert. Die universitäre Kultur des 13. und 14. Jahrhunderts könne somit als antirhetorisch beschrieben werden. Obwohl mehr als 100 mittelalterliche Handschriften der aristotelischen ,Rhetorik ${ }^{6}$ überliefert sind ${ }^{11}$, wurde dieses Werk im universitären Milieu vernachlässigt ${ }^{12}$. Die soziokulturelle Realität spiegelt diese antirhetorische Einstellung wider. Nach 1220 taucht der rhetorische Unterricht in den Ausbildungs- und Prüfungsordnungen der europäischen Universitäten kaum auf. Die Satzungen der Universität Paris von 1215 erwähnen

10 McLuhan, The Classical Trivium (nt. 7), 17: ,[...] Aristotle established the nature of non-grammatical scientific method in the Posterior Analytics. His achievement bore no fruit until the twelfth century. Until the twelfth century, therefore, grammar reigned unrivalled as the prime mode of science, and, from the patristic period, of theology as well. [...] Classical scholars have been indifferent to the extraordinary role of classical grammar in medieval science and theology, and, therefore, confused about the nature of the Renaissance.“ Ibid., 175: „However, enough evidence has already been adduced to show that 'scholastic culture' is abstracted from the Middle Ages when we so speak. It was only one aspect of the culture of those centuries, and was heartily opposed by the more typical patristic-classical culture which had long been in vogue and which suffered only a temporary eclipse from the dialecticians."

11 James Murphy zählt 22 Handschriften des 13. Jahrhunderts, 57 Handschriften des 14. Jahrhunderts und 17 Handschriften des 15. Jahrhunderts (J. J. Murphy, Rhetoric in the Middle Ages. A History of Rhetorical Theory from Saint Augustine to the Renaissance, Berkeley - Los Angeles London 1974, 97). Im Band 31/1-2 des Aristoteles Latinus (Rhetorica. Translatio Anonyma sive Vetus et Translatio Guillelmi de Moerbeka, ed. B. Schneider, Leiden 1978) erwähnt B. Schneider 4 Handschriften für die Translatio Anonyma und 99 Handschriften, die die Übersetzung Wilhelms von Moerbeke enthalten.

12 Die Verweise auf die aristotelische ,Rhetorik' in scholastischen Werken des 13./14. Jahrhunderts sind im Vergleich mit der Verwendung anderer aristotelischer Werke selten. Dieselbe Feststellung gilt für die Kommentare zur ,Rhetorik'. Im 13. Jahrhundert wurden wahrscheinlich nur drei Kommentare zur ,Rhetorik' verfasst: diejenigen von Albert dem Großen, Ägidius von Rom und Boethius von Dacien (cf. C. Marmo, L’utilizzazione delle traduzioni latine della Retorica nel commento di Egidio Romano [1272-1273], in: G. Dahan/I. Rosier-Catach [eds.], La Rhétorique d'Aristote. Traditions et commentaires de l'Antiquité au XVII ${ }^{\mathrm{e}}$ siècle [Tradition de la pensée classique], Paris 1998, 111-134). James Murphy schließt daraus, dass das Trivium während der Scholastik nicht wirklich existierte: „Apparently he [the student] did not undergo a course of rhetoric at all, so that the popular conception of a regular trivium at the university level is largely a myth." (Murphy, Rhetoric [nt. 11], 105.) 
die Rhetorik zwar, aber als sekundär ${ }^{13}$. Danach tritt sie in den Statuten der Universitäten von Paris und Oxford bis 1458 nicht mehr in Erscheinung ${ }^{14}$.

Diese neue Konzeption des Wissens und der Lehre bedurfte aber auch theoretischer Legitimierung und setzte sich nicht reibungslos durch. Ich werde hier drei häufig vernachlässigte Aspekte dieser antirhetorischen Strategie skizzenhaft behandeln. Meiner Einschätzung nach sollte die Scholastik eher als Chiffre für eine Institution denn als ein Zeitalter betrachtet werden. Diese Institution, die Universität, beruht erstens auf einer in den Diskursen der Kleriker konstruierten Auseinandersetzung zwischen Wissenschaftlichkeit und Öffentlichkeit. Zweitens hat das scholastische Latein eine bedeutende Rolle bei der Entstehung und Durchsetzung des scholastischen Habitus gespielt. Drittens ist das scholastische Latein eng mit Prozessen der Verschriftlichung wissenschaftlicher Praktiken verbunden, die der Redekunst entgegengestellt wurden.

\section{Wissenschaftlichkeit gegen Öffentlichkeit}

Zum einen kann also eine Trennung von Wissensbereichen im 13. Jahrhundert festgestellt werden. Die theoretischen Wissenschaften, insbesondere die Naturphilosophie, die Metaphysik und die spekulative Theologie gehörten fast ausschließlich zur Universität. Sie bildeten einen geschlossenen wissenschaftlichen Raum, dessen intellektuelle Akteure, die zum Klerus ${ }^{15}$ gehörten, einen exklusiven Anspruch auf die Wissenschaftlichkeit erhoben ${ }^{16}$. Diese klerikale Wissenschaftlichkeit grenzte sich von den weltlichen Wissensformen ab, die für die

${ }^{13}$ H. Denifle/A. Chatelain (eds.), Chartularium Universitatis Parisiensis, 4 vols., Paris 1889-1897 [Neudruck Bruxelles 1964], vol. 1, 78 (n. 20): „Non legant in festivis diebus nisi philosophos et rhetoricas, et quadruvialia $[\ldots]^{\prime \prime}$.

14 I. Hajnal, L’enseignement de l'écriture aux universités médiévales, Budapest 1959, 159 sqq.; Murphy, Rhetoric (nt. 11), 57-101.

15 Zur Bedeutung dieses Konzepts in diesem geschichtlichen und institutionellen Zusammenhang cf.: H. Grundmann, Sacerdotium - Regnum - Studium. Zur Wertung der Wissenschaft im 13. Jahrhundert, in: id., Ausgewählte Aufsätze, vol. 3 (Monumenta Germaniae Historica. Schriften 25/3), Stuttgart 1978, 275-291; H. Grundmann, Vom Ursprung der Universität im Mittelalter, ibid., 292-342; Y. Congar, Clercs et laïcs au point de vue de la culture au moyen âge: «laicus» = «sans Lettres», in: Studia mediaevalia et mariologica. P. Carolo Balić OFM septuagesimum explenti annum dicata, Roma 1971, 309-332; S. Lusignan, "Vérité garde le roy». La construction d'une identité universitaire en France (xIII ${ }^{\mathrm{e}}-\mathrm{xv}^{\mathrm{e}}$ siècle), Paris 1999, 102-122, 177; R. Imbach/C. König-Pralong, Le défi laïque. Existe-t-il une philosophie de laïcs au Moyen Âge? (Conférence Pierre Abélard), Paris 2013, 33-50.

16 In diesem Zusammenhang ist anzumerken, dass das mittelalterliche „Lateineuropa“ hinsichtlich der Weitergabe gelehrten Wissens eine Ausnahme darstellt. Hier kompensierten und ersetzten neue Institutionen wie Universitäten und Kathedralschulen das schwache „Verwandtschaftskoordinatensystem“, das für die Wissensvermittlung durch Erbschaft nicht mehr hinreichend war. Cf. G. Algazi, Habitus, familia und formae vitae: die Lebensweisen mittelalterlicher Gelehrten in muslimischen, christlichen und jüdischen Gemeinden - vergleichend betrachtet, in: F. Rexroth (ed.), Beiträge zur Kulturgeschichte der Gelehrten im Mittelalter (Vorträge und Forschungen. Konstanzer Arbeitskreis für mittelalterliche Geschichte 73), Ostfildern 2010, 185-217. 
Öffentlichkeit geeignet konzipiert und in dieser auch tatsächlich praktiziert wurden. Dabei gebrauche ich den Begriff „Öffentlichkeit“ nicht im spezialisierten Sinne, den ihm Habermas ursprünglich verliehen hat, sondern als Synonym von weltlicher oder adeliger Gesellschaft.

Die mittelalterliche Öffentlichkeit ist das Reich der ciceronianischen Rhetorik par excellence. Ein Vehikel der ciceronianischen rhetorischen Lehre seit der Antike ist die Literaturgattung der Fürstenspiegel; darüber hinaus wurden die beiden Cicero zugeschriebenen Schriften über die Rhetorik, die ,Rhetorica ad Herennium' und der Traktat ,De inventione', mehrfach ins Deutsche, Französische und Italienische übersetz $\mathrm{t}^{17}$. In seiner kommentierten Übersetzung der ,Rhetorica ad Herennium“ (1261/62) schreibt Brunetto Latini der Rhetorik zwei Merkmale zu. Ihm zufolge zielt sie auf die Beredsamkeit ab, da sie auf die mündliche Kommunikation ausgerichtet ist, und sie dient der Politik, dem Regieren der weltlichen Gesellschaft: „Adonque la scienza del covernamento delle cittadi è cosa generale sotto la quale si comprende rettorica, cioè l'arte del bene parlare." 18

In der zweiten Hälfte des 13. Jahrhunderts nun, im universitären Milieu, wurde gerade diese Wissensform bewusst und stark abgewertet. Die Doktoren begründeten die Autonomie und die Überlegenheit des scholastischen Lehrens und Lernens theoretisch, indem sie die Wissenschaftlichkeit wie einen geschlossenen Raum vom rhetorischen Wissen abgrenzten. Thomas von Aquin nimmt bedenkenlos an, dass die Rhetorik kein Teil der Theologie ist: „Rhetorica autem non est pars theologiae" (ST I-II, q. 7, art. 2, arg. 3). Diese Auffassung ist bemerkenswert, wenn sie in der longue durée der Theologiegeschichte betrachtet wird. Bis zum 12. Jahrhundert wurde nämlich die Theologie mit der Bibelexegese identifiziert und eng mit der Grammatik und der Rhetorik verbunden. Im 14. Jahrhundert bezeugen repräsentative Bibelkommentare, u. a. diejenigen von Nikolaus von Lyra, Nikolaus Trevet und Robert Holcot, das Fortdauern der wörtlichen, mit der Grammatik und der Rhetorik zusammenhängenden Exegese.

17 Zur französischen Tradition: J. Monfrin, Humanisme et traductions au Moyen Âge, in: Journal des savants (1963), 161-190, insb. 168-170; L. Delisle, Maitre Jean d'Antioche, traducteur, et frère Guillaume de Saint-Etienne, in: Histoire littéraire de la France 33 (1906), 1-40; R. H. Lucas, Mediaeval French Translations of the Latin Classics to 1500, in: Speculum 45 (1970), 225-253, hier 235-237. Zu Brunetto Latini und dessen Übersetzung der ,Rhetorica ad Herennium (La Rettorica)': Imbach/König-Pralong, Le défi laïque (nt. 15), 77-81. Im italienischsprachigen Raum ist auch der ,Fiore di rettorica' des Bruders Guidotto da Bologna nennenswert. Cf. S. Segre, Les formes et traditions didactiques, in: H. R. Jauss (ed.), Grundriß der romanischen Literaturen, vol. 6/2, Heidelberg 1970, 173-174 (n. 3408). Zur deutschen Tradition: P. Kesting, Cicero, in: Verfasserlexikon, vol. 1, 1279-1282. Die Schriften ,De officiis‘, ,De amicitia und ,De senectute‘ wurden ebenfalls ins Französische übersetzt. Cf. C. Galderisi (ed.), Translations médiévales. Cinq siècles de traductions en français au Moyen $\hat{A} g e\left(\mathrm{XI}^{\mathrm{e}}-\mathrm{XV}^{\mathrm{e}}\right.$ siècles). Étude et Répertoire, 2 vols., Turnhout 2011; P. Chavy, Traducteurs d'autrefois, Moyen âge et Renaissance. Dictionnaire des traducteurs et de la littérature traduite en ancien et moyen français, 842-1600, Paris-Genève 1988.

18 Brunetto Latini, La rettorica, ed. F. Maggini, Firenze 1915, 29, 4. 
Die wissenschaftlichen Unternehmen dieser Theologen wurden in bewusster Auseinandersetzung mit der rein spekulativen Theologie konzipiert und ausdrücklich gerechtfertigt.

Die Werke der beiden Theologen des 13. Jahrhunderts, die einen Kommentar zur aristotelischen ,Rhetorik' verfasst haben, Albert der Große und Ägidius von Rom ${ }^{19}$, weisen hingegen sehr abwertende Konzeptionen der Rhetorik auf. In seinem Metaphysikkommentar (nach 1263) schließt Albert die Rhetorik aus dem Bereich der Wissenschaftlichkeit aus. Das Gehirn des Redners und Rhetoriklehrers sei so „gefroren“, dass dieser überhaupt keinen Zugang zur Spekulation habe: "Quorum autem congelati sunt spiritus et non bene clari propter frigus inspissans, occupantur circa signa rhetorica et detinentur in his nec profundantur in aliqua veri speculatione. $“ 20$

Ägidius von Rom konzipiert die Disziplin als ein für die Laien geeignetes Wissen, das den universitären Standards nicht entspreche. Im Traktat ,De regi-

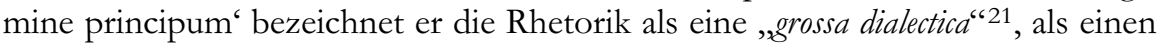
groben Ersatz der Dialektik, während er im Rhetorikkommentar (1272/73) seine Adressaten als einfache und ungehobelte Menschen beschreibt. Auf diese Weise trennt er deutlich zwei Wissensbereiche: „Tertia est quia iudex locutionis rhetoricae et eius auditor est simplex et grossus; anditor vero locutionis dialecticae babet esse ingeniosus et subtilis.“"22

In rein scholastischen Werken trifft der Philosophiehistoriker oft solche herablassenden Bewertungen der öffentlichen Gesellschaft an, in Schriften also, die von vornherein nichts mit Laien zu tun haben. Unter zahlreichen Beispielen ${ }^{23}$ ist in dieser Hinsicht der Fall von Roger Bacon besonders interessant. Bekanntlich hat Roger Bacon die Universität Paris und ihre wissenschaftlichen Praktiken

19 Zum Kommentar des Ägidius: Marmo, L’utilizzazione (nt. 12). Zum verlorenen Kommentar Alberts: C. Lohr, Medieval Latin Aristotle Commentaries, in: Traditio 23 (1967), 313-413, hier 345.

20 Albert der Große, Metaphysica, I, 1, 5, ed. B. Geyer (Opera omnia 16/1), Münster 1960, 8, 24-28. Die Theorie der Flüssigkeiten und Komplexionen des Gehirns wird von Albert im Traktat ,De natura loci‘ (Tract. 2, cap. 3) erörtert.

21 Ägidius von Rom, De regimine principum, II, 2, 8, Romae 1656, 182v. Dieser Philipp dem Schönen gewidmete Fürstenspiegel entstand vor 1282.

22 Ägidius von Rom, Rhetorica Aristotelis cum fundatissimi arcium et theologiae doctoris Egidii de Roma luculentissimis commentariis, Venetiis 1515, $1^{\text {va }}$. Siehe auch: G. Bruni, The De differentia Rhetoricae, Ethicae et Politicae of Aegidius Romanus, in: The New Scholasticism 6 (1932), 1-18. Zu Ägidius' Bewertung der Rhetorik: S. Robert, Rhetoric and Dialectic according to the first Latin commentary on the Rhetoric of Aristotle, in: The New Scholasticism 31 (1957), 484-498; J. J. Murphy, The Scholastic Condemnation of Rhetoric in the Commentary of Giles of Rome on the Rhetoric of Aristotle, in: Arts libéraux et philosophie au Moyen Âge. IV ${ }^{\mathrm{e}}$ Congrès international de philosophie médiévale, Montréal-Paris 1969, 833-841. Zum Rhetorikunterricht im Mittelalter: Murphy, Rhetoric (nt. 11); K. M. Fredborg, The Scholastic Teaching of Rhetoric in the Middle Ages, in: Cahiers de l'Institut du moyen âge grec et latin 55 (1987), 85-105.

23 Cf. C. König-Pralong, Le bon usage des savoirs. Scolastique, philosophie et politique culturelle (Études de philosophie médiévale 98), Paris 2011, 29-104. 
grundsätzlich und heftig kritisiert ${ }^{24}$. Er stellt aber nicht die Geschlossenheit der universitären Wissenschaftlichkeit in Frage. Im Gegenteil: Er drängt darauf, den einfachen Menschen naturphilosophische Kenntnisse vorzuenthalten. So rät er bei der Erörterung des Zustands der Tierseele nachdrücklich davon ab, den Laien dieses Wissen zu vermitteln. Ihrer Unwissenheit wegen würden die Laien über die Kleriker spotten: „Immo vulgus laicorum in multis regnis adbuc credit quod soli bomines animas babent, unde derident clericos qui dicunt canes et cetera bruta babere animas $[\ldots] . “ 25$

Dieses wissenschaftliche Überlegenheitsgefühl beruht auf einer theoretisch begründeten Konzeption des Wissens als einer von den politischen Instanzen unabhängigen symbolischen Macht. In seinem dritten ,Quodlibet' (1270) begründet beispielsweise Thomas von Aquin die Autonomie der universitären Lehre gegenüber dem auf das Regieren ausgerichteten Wissen theoretisch. In Sonderfällen könne ein Bakkalaureus aus sich selbst heraus Anspruch auf den Doktortitel, die „licentia docendi“, erheben, da die dafür erforderlichen spekulativen Kenntnisse immanent sind. Diese Kenntnisse werden subjektiv erworben und vom Subjekt besessen. Anders also als beispielsweise die bischöfliche Macht, die wie alle anderen politischen Mächte von außen verliehen wird und von einer sozialen Instanz abhängt, könnte sich die symbolische Macht des Theologiemagisters somit theoretisch jeder sozialen Kontrolle entziehen:

„Secunda differencia est, quod eminencia sciencie, que requiritur ad cathedram magistralem, est perfectio hominis secundum se ipsum; eminencia uero potestatis, que pertinet ad cathedram pontificalem, est hominis per comparationem ad alium." 26

Letztendlich setzt diese von den Magistern inszenierte Trennung der universitären Lehre von der Gesellschaft und dem weltlichen Wissen unterschiedliche Haltungen gegenüber dem Wissen voraus. Die Adligen bedienen sich der Rhetorik und der Politik, um zu regieren. Sie bringen kein neues Wissen hervor. Die Magister müssen dagegen von ihren Schülern als Autoren und Autoritäten anerkannt werden. Sie tragen zur Entwicklung des Wissens bei. Dem berühmten Magister Heinrich von Gent zufolge ist die Wahrheit auf die Person des Magisters zurückzuführen. Die Autorität des Dozierenden ist wichtiger und zuverlässiger als der Inhalt seiner Lehre; sie stellt das letzte Kriterium der Wahrheit dar. Als Person ist der Theologiemagister ein Garant der Wahrheit, als ob der Heilige Geist durch ihn spräche. Um die Autorität der Theologiemagister zu verteidigen, betont Heinrich deren intellektuelle Überlegenheit, die sie vor jedem persönlichen Angriff bewahren sollte:

„[...] quasi nibil esset quod dixerat, et quasi magist $\langle$ er $\rangle$ theologiae ex hoc quod est magister et sedens in cathedra nullam omnino baberet auctoritatem, et non esset ei ex boc credendum in aliquo

24 Cf. König-Pralong, Le bon usage des savoirs (nt. 23), 128-164.

25 Roger Bacon, Liber primus Communium naturalium, Pars Quarta, Distinctio tertia de anima, ed. R. Steele (Opera hactenus inedita Rogeri Baconi Fasc. III), Oxford 1911, 283.

26 Thomas von Aquin, Quodlibet III, q. 4, art. 1 (,Utrum liceat quod aliquis pro se petat licenciam in theologia docendi'), ed. Commissio Leonina (Opera omnia 25/2), Roma-Paris 1996, 252 $2^{\text {b }}$ 
quod est magister et sedens in cathedra, quod non est verum. [...] Unde et si aliquando magister dicat aliquid quod nec auctoritate alia nec ratione confirmat, bene tamen quoquo modo ei ex sola auctoritate propria magisterii credendum est, et si nibil obstet, tenendum est quod illud pertineat ad cathedram et quod Spiritus sanctus illud tamquam verum loquatur in ipso. Non enim solum spectat ad cathedram quod expressum est in lege divina et per sanctos expositum, sed etiam illud quod usque in finem mundi per veridicos doctores certa ratione restat explicandum. Et sic in magistro theologiae attendendum est non tam quid dicat, sed quis dicat $[\ldots] .{ }^{\text {" } 27}$

\section{Das scholastische Latein}

Mittels seines spekulativen Lehrprogramms und durch die Selbstlegitimierung der Magister hat sich das universitäre Wissen von den angewandten Wissenschaften abgegrenzt. Das universitäre Wissen beruht darüber hinaus auf einer gewissen technischen Kompetenz, dem scholastischen Latein. Dieses Idiom ist das zweite Merkmal der universitären antirhetorischen Wissenskonzeption, das ich hier hervorheben möchte. Das universitäre Wissen hängt nämlich mit dem Gebrauch eines Idioms zusammen, das seinerzeit niemand mehr als Muttersprache beherrscht und gelernt hat. Latein war nicht länger eine einheimische Sprache, mindestens seit dem Anfang des 8. Jahrhunderts. Die Sprachwissenschaftler und Medienhistoriker bezeichnen diese kulturelle Besonderheit als Diglossie ${ }^{28}$. Neben ihrer jeweiligen alltäglichen Kommunikationssprache lernten die Schüler und Magister der mittelalterlichen Universität eine technische Sprache, die außerhalb der Mauern der Universität sehr selten gesprochen wurde. Alle Texte, die für den Unterricht vorgesehen waren, wurden in diesem Idiom gelesen, was bedeutet, dass sie gegebenenfalls auch ins Lateinische übersetzt werden mussten. Darüber hinaus hing das scholastische Latein von einer Schriftkultur ab: Wer Latein sprechen konnte, war auch in der Lage es zu lesen. Wer Latein beherrschte, hatte es durch Lesen und Schreiben gelernt. Walter Ong und Michael Clanchy beschreiben das scholastische Latein als „a textualized language“, als eine von Texten abhängige Sprache, die durch die Regeln des Schreibens wesentlich bestimmt wird ${ }^{29}$. Bemerkenswerterweise hob schon Dante Alighieri in einem berühmten Kapitel seines Traktats ,De vulgari eloquentia‘ (ca. 1305) alle

${ }^{27}$ Heinrich von Gent, Tractatus super facto praelatorum et fratrum, edd. L. Hödl/M. Haverals (Opera omnia 17), Leuven 1989, 131.

28 Cf. W. J. Ong, Orality, Literacy, and Medieval Textualisation, in: New Literary History 16 (1984), 1-12, hier 4. Zum Latein als gelehrter Weltsprache: J. Leonhardt, Latein: Geschichte einer Weltsprache, München 2008.

29 Ong, Orality, Literacy (nt. 28), 7: „No one could speak it who could not write it and who had not learned to speak it through writing it. It was a chirographically controlled language, or, as we may put it, a textualized language." Siehe auch: W. J. Ong, Orality and Literacy. The Technologizing of the Word, London-New York 1982, 110-113; sowie die anregende und viel umfangreichere Studie von M. T. Clanchy, From Memory to Written Record. England 1066-1307, Malden-Oxford 1979, ${ }^{2} 1993$. 
diese Eigenschaften des scholastischen Lateins hervor. Er nannte es „Grammatik“:

„Est et inde alia locutio secundaria nobis, quam Romani gramaticam vocaverunt. [...] ad habitum vero buius pauci perveniunt, quia non nisi per spatium temporis et studii assiduitatem regulamur et doctrinamur in illa." $" 30$

Als nicht- oder semi-Scholastiker konnte Dante die Eigentümlichkeit dieses Idioms besonders gut, gleichsam von außen her beobachten. Aber auch einige Theologiemagister waren sich dessen bewusst, dass ihre wissenschaftliche Sprache außergewöhnlich war. Im Gegensatz zu Dante aber priesen diese Magister die Überlegenheit des scholastischen Lateins als wissenschaftliches Idiom. Ich denke namentlich an den Franziskaner Johannes Peckham, der zwischen 1270 und 1272 an der Universität Paris als Theologiemagister unterrichtete. Peckham stellt eine sehr interessante linguistische Hypothese auf. Diese lautet: Möglicherweise werden wir auch im Paradies eine Sprache sprechen, damit unsere Sprachorgane nicht vergebens auferstehen. Dabei könnte es sich gut und gerne um das moderne scholastische Latein handeln:

„Ad tertium dicendum quod ibi erit forte aliqua lingua nova conveniens statui gloriae. Et si ibi futura sit aliqua lingua modernorum, puto quod erit mere latina. [...] Et ita forte aeternaliter remanebit, nec in lingua latina vulgari et corrupta, quia nibil corruptum est ibi.“31

Laut Peckham könnte das moderne Latein also die ewige und reine, d. h. nicht einheimische, Sprache im Paradies sein. Damit befreit Peckham das sprachliche Werkzeug des scholastischen Studiums von der weltlichen Kontingenz und der gesellschaftlichen Vergänglichkeit. Dreißig Jahre später wird Dante die Überlegenheit der Volkssprache aus genau entgegengesetzten Gründen verteidigen. Dante zufolge ist die Volksprache überlegen, gerade weil sie, wie der Mensch und das irdische Leben, vergänglich ist.

\section{Prozess der Verschriftlichung}

Die dritte Bedingung der universitären mittelalterlichen Kultur, die zu antirhetorischen Haltungen und Stellungnahmen beitrug, ist die massive Verschriftlichung des Wissens. Das alte gängige Vorurteil, demgemäß die mittelalterliche

30 Dante Alighieri, De vulgari eloquentia, i, 3. Übersetzt von F. Cheneval, mit einer Einleitung von R. Imbach und I. Rosier-Catach und einem Kommentar von R. Imbach und T. Suarez-Nani, Hamburg 2007, 2-4.

31 Johannes Peckham, Quaestiones de beatitudine animae et corporis, q. 8 (,Utrum in patria omnes sensus sint in actibus suis), in: id., Quaestiones disputatae, edd. G. J. Etzkorn/H. Spettmann/ L. Oliger, Grottaferrata 2002, 538. 
Kultur eine wesentlich mündliche Kultur gewesen sei $^{32}$, wurde schon vor einigen Jahrzehnten von der Mittelalterforschung kritisiert und zurückgewiesen ${ }^{33}$.

Zwei Wissensformen veranschaulichen besonders gut die Bedeutung dieses Prozesses der Verschriftlichung: die ars dictaminis und die ars praedicandi. Diese Wissensformen waren keine Wissenschaften sondern Künste, die aber zu praktischen Zwecken an der Universität unterrichtet wurden. In dieser Hinsicht bildeten sie eine Zwischenwelt, sie standen zwischen Öffentlichkeit und universitärer Wissenschaftlichkeit. Beide waren mit der Rhetorik insofern verbunden, als sie sozialen Interessen dienten. Beide wiesen jedoch wichtige Prozesse der Verschriftlichung und der institutionellen Regulierung auf, die mit einer graduellen Entfremdung von der Beredsamkeit zusammenhingen. Die ars dictaminis wurde insbesondere an den italienischen Universitäten unterrichtet ${ }^{34}$. Diese technische Kompetenz war bis zum 15. Jahrhundert die einzige Form rhetorischen Wissens, die an der Universität gelehrt wurde. Sie hatte aber mit der ciceronianischen Beredsamkeit nichts zu tun. Als Kunst der notariellen Praxis diente sie dem Verfassen von offiziellen Dokumenten und war der mündlichen Beredsamkeit fremd ${ }^{35}$.

Dasselbe Verschwinden der Redekunst lässt sich im Fall der ars praedicandi belegen. Diese Literaturgattung, die im 13. Jahrhundert aufblühte, zeichnet sich durch eine regelrechte Distanzierung von der Beredsamkeit aus ${ }^{36}$. Nicole Bériou hat die Vorbehalte der Verfasser solcher Artes gegen das freie Predigen stu$\operatorname{diert}^{37}$. Sie zeigt in diesem Zusammenhang, dass die Predigthandbücher kein Interesse für die rhetorische Gestalt und den Stil aufweisen, sondern auf die Inhalte der Predigten ausgerichtet waren. Als Kritiker der Scholastik seiner Zeit stellte selbst Roger Bacon dieses kulturelle Phänomen fest und tadelte es: „Quae forma praedicandi [i.e. ars eloquendi] non tenetur a vulgo theologorum, sed sunt elongati ab ea his diebus.“ 38

32 Walter J. Ong teilt noch diese zu pauschale Ansicht, wenn er schreibt: „But for all their textuality, medieval universities were radically oral as universities today no longer are.“ (Ong, Orality [nt. 28], 3).

${ }^{33}$ Für die weltliche Kultur ist die Studie von Michael T. Clanchy ausschlaggebend (Clanchy, From Memory to Written Record [nt. 29]). In Deutschland wurden zwei Sonderforschungsbereiche diesem Thema gewidmet: SFB 231 (Universität Münster), ,Träger, Felder, Formen pragmatischer Schriftlichkeit im Mittelalter“; SFB 321 (Universität Freiburg), „Übergänge und Spannungsfelder zwischen Mündlichkeit und Schriftlichkeit “. Für eine Darstellung der neueren Forschung, siehe: P. Chastang, L’archéologie du texte médiéval. Autour de travaux récents sur l'écrit au Moyen Âge, in: Annales HSS 63 (2008), 245-269. Siehe auch: P. Porro, Dalla Pagina alla scientia. L'identificazione tra libri e sapere scientifico nel medioevo scolastico e il caso anomalo della teologia, in: Quaestio 11 (2002), 225-254.

34 Hajnal, L'enseignement de l'écriture (nt. 14), 159 sqq.; Murphy, Rhetoric (nt. 11), 194-268.

35 Cf. die umfassende Studie von M. Camargo, Ars dictaminis, Ars dictandi (Typologie des sources du Moyen Âge occidental 60), Turnhout 1991.

36 Cf. Murphy, Rhetoric (nt. 11), 269-356.

37 N. Bériou, L'avènement des maîtres de la parole. La prédication à Paris au XIII ${ }^{\mathrm{e}}$ siècle, 2 vols., Paris 1998, vol. 1, 145-156.

38 Roger Bacon, Opus tertium, cap. 75, in: Opera quaedam hactenus inedita, ed. S. J. Brewer, London 1859, 309. 
In diesem Zusammenhang geht die heutige Mittelalterforschung noch einen Schritt weiter und zieht den angeblich mündlichen Charakter der rein universitären Literaturgattungen, der Streitfragen, Sentenzenkommentare, universitären Predigten und Kommentare, insgesamt in Zweifel. In einem noch nicht veröffentlichten, im Juli 2014 gehaltenen Vortrag präsentierte Loris Sturlese die Predigten Meister Eckharts als ein wesentlich schriftlich verfasstes Werk. Ähnlich weisen die universitären reportationes sehr oft wichtige, auf die Schriftlichkeit als primäres Medium hinweisende Revisionen auf. Beispielsweise bezieht sich der Verfasser auf weitere Textstellen. Die Sentenzenkommentare schließlich bilden geradezu den Inbegriff für diese Prozesse schriftlicher Überarbeitung. Ein paradigmatisches Beispiel hierfür bietet der Sentenzenkommentar des Durandus von St. Pourçain, der am Thomas-Institut der Universität zu Köln ediert wird ${ }^{39}$. Die überlieferten Handschriften bezeugen drei aufeinanderfolgende Redaktionen. Sie zeigen die unermüdliche Arbeit des Magisters, der seinen Text ständig revidiert hat. Diese Sorge des Magisters, die Verbreitung seines Werkes zu kontrollieren, ist keine Besonderheit des 14. Jahrhunderts. Schon gegen 1278 bedauerte beispielsweise Johannes Peckham die ungeregelte Verbreitung seiner ,Perspectiva in einer nichtrevidierten Redaktion:

„Scripsi dudum rogatus a sociis quedam mathematice ruditer rudimenta, que tamen aliis occupatus incorrecta reliqui, que etiam contra intentionem meam in publicum prodierunt."40

Der scholastische Magister ist nicht nur ein Lehrer, sondern auch ein Schriftsteller. Er konzipiert sich selbst als Autor eines Werkes. Ich kann hier nicht in die Details gehen und verweise daher nur auf die Prologe der beiden Summen des Thomas von Aquin, die deutlich ein schriftstellerisches Selbstbewusstsein belegen. Dasselbe gilt für die sorgfältig revidierte Summe des Heinrich von Gent. Der Fall des Dietrich von Freiberg ist noch offensichtlicher. Dietrich hat klar strukturierte und thematisch gut definierte Traktate verfasst, deren Prologe häufig die Absicht des Autors erklären und präzisieren ${ }^{41}$. Schriftkultur und Kontrolle über die Verbreitung der Werke kennzeichnen das scholastische Wissen und grenzen dieses von der Redekunst und der öffentlichen Beredsamkeit ab.

V. Schluss: Kritiken des scholastischen antirhetorischen Modells

Das wissenschaftliche Ideal eines antirhetorischen Stils ist wohl mit der Universität im 13. Jahrhundert entstanden. Es bildet ein Moment der Bildungsge-

39 Cf. A. Speer/F. Retucci/T. Jeschke/G. Guldentops (eds.), Durand of Saint-Pourçain and His Sentences Commentary. Historical, Philosophical, and Theological Issues (Recherches de Théologie et Philosophie Médiévales. Bibliotheca 9), Leuven-Paris - Walpole 2014.

40 John Pecham and the Science of Optics. Perspectiva Communis, ed. with an introd., English transl., and critical notes by D. C. Lindberg, Madison 1970, 60.

41 Cf. König-Pralong, Le bon usage des savoirs (nt. 23), 69-104 (zu Heinrich von Gent), $216-$ 278 (zu Dietrich von Freiberg). 
schichte, dessen mächtige Nachklänge noch lange im Bereich des abendländischen universitären Wissens nachweisbar sind. Dieses an der mittelalterlichen Universität formulierte, im 19. Jahrhundert von Renan gelobte und heute noch von den meisten Akademikern vertretene Wissensideal setzt eine Abgrenzung der Wissenschaftlichkeit von der Öffentlichkeit voraus. Im Mittelalter stützte sich diese Wissensform auf den Gebrauch einer spezialisierten technischen Sprache, des scholastischen Lateins, und sie verwirklichte sich als schriftliches, institutionell konstituiertes Wissen.

Bereits die mittelalterlichen Kritiken des scholastischen Wissens hatten die drei hier dargestellten Merkmale identifiziert. Innerhalb der scholastischen Welt war wahrscheinlich Roger Bacon der größte Gegner der scholastischen Prakti$k^{4}{ }^{42}$. Er warf den Doktoren seiner Zeit vor, die Moralphilosophie und die Rhetorik zu vernachlässigen, sich selbst als Autoren und Autoritäten zu konzipieren, alle anderen Sprachen außer dem barbarischen scholastischen Latein zu ignorieren und zu viel zu schreiben statt zu predigen. Innerhalb des Dominikanerordens betonte Humbertus de Romanis die Wichtigkeit des Predigens gegen die spekulative Philosophie ${ }^{43}$ : „Alii sunt qui libenter vacant lectionibus sacris: sed buiusmodi studium non dirigitur ad doctrinam praedicationis, quae utilitas in illo? " 44

Trotz dieser Besonderheiten darf die Scholastik nicht einfach schlechthin mit einer spekulativen, antirhetorischen und antiliterarischen Wissensform identifiziert werden. Für die Exemplasammlungen des Johannes von Wales beispielsweise sind antike Rhetoriker wie Cicero und Seneca von höchster Bedeutung. Am Anfang des 14. Jahrhunderts blühte der wörtliche und literarische Kommentar der Bibel und anderer Hauptwerke der christlichen Gelehrsamkeit wie des ,Gottesstaates' von Augustin wieder auf. Nikolaus von Lyra, Nikolaus Trevet, die classici von Oxford verteidigten nicht-spekulative Konzeptionen der Theologie, die auf das Predigen ausgerichtet waren und zum Teil auch weltlichen Mächten dienten.

Außerhalb der Universität ${ }^{45}$ kritisierte Petrarca grundlegend die Scholastik. Sein ganzes Werk, das die Rhetorik ins Zentrum der Gelehrsamkeit rückt, kann als eine Widerlegung der scholastischen Wissenskonzeption gelesen werden. Ich schließe diese knappen Überlegungen aber mit dem Zeugnis von Boccaccio. Im 14. Buch seiner, Genealogia deorum gentilium'verteidigt Boccaccio die Dichtkunst, die Grammatik und die Rhetorik gegen deren Gegner, namentlich gegen die Theologiemagister. Interessanterweise antizipiert er das Urteil und die Feindlichkeit der Theologiemagister gegen sich selbst: „Ex quibus fatuitatibus iam satis scio quid monstrum istud spectantes in me, quid in opus meum, quid in Poëtas dicturi

42 Cf. König-Pralong, Le bon usage des savoirs (nt. 23), 128-164.

43 Cf. von Moos, Rhetorik, Dialektik (nt. 7), 147.

44 Umbertus de Romanis, De eruditione praedicatorum, I, 20, Barcinonae 1607, 442 ${ }^{\mathrm{aD}}$.

45 Frank Rexroth hat darüber hinaus die zunehmenden Vorbehalte und Kritiken gegen die universitäre Expertenkultur studiert. Cf. u. a. F. Rexroth, Expertenweisheit. Die Kritik an den Studierten und die Utopie einer geheilten Gesellschaft im späten Mittelalter (Freiburger mediävistische Vorträge 1), Basel 2008. 
sunt."46 Die Theologiemagister vertrieben nicht nur die Grammatik und die Rhetorik aus der Universität; sie fungieren auch als deren Feinde außerhalb der Universität, im öffentlichen Raum.

46 Giovanni Boccaccio, Genealogia deorum gentilium, XIV, 3, Basileae 1532, 354. 